\title{
Muscular Dystrophy during Osteoarthritic Disorder: A Novel Diagnostic Protocol Evidenced by Biochemical and Anatomical
}

\section{Parameters}

\begin{abstract}
Background: Muscular Dystrophy during Osteoarthritic Disorder (MD-OAD) is a group of disorders characterized by muscle wasting weakness and degeneration along with potential loss of joint cartilage, bone hypertrophy, painful Joint effusion and restricted movement of joints. Creatine Kinase-Muscle (CK-MM) and AldolaseA (AldoA) are considered to be realistic biomarkers to detect the MD-OAD. The aim of the study was to evaluate the serum levels of CK-MM and AldoA and determine their correlation with risk factors such as muscle weakness, muscle degeneration, skeletal muscle damage resulting in deranged anatomical features developed in MD-OAD.
\end{abstract}

Methods: Baseline data from 162 patients, aged $59.89 \pm 7.19$ years, suffering with MD-OADs for $7.89 \pm 1.90$ years and 153 participants, aged $58.96 \pm 8.63$ years, without MD-OADs were collected in this cross sectional study. Separate analyses were performed for participants with and without MD-OAD symptoms confirming with electromyography, musculoskeletal ultrasound, muscle biopsy, CT-scan and MRI. Serum CK-MM and AldoA levels were estimated. All subjects, with and without MD-OADs, underwent standardized physical, radiographic, other related examinations and completed a questionnaire.

Results: The levels of CK-MM and AldoA of experimental subjects, representing the risk factor of $M D-O A D s$, were increased their mean $\pm S D$ values $248.42 \pm 100.96$ and $8.87 \pm 2.70 \mathrm{U} / \mathrm{L}$ respectively and abnormal anatomical features, pain syndromes and obesity were all exhibited highly significant differences $(p<0.0001)$ when compared with the control subjects.

Conclusion: It is firmly concluded that monitoring the levels of serum CK-MM and AldoA along with anatomical parameters features, pain free life-style and body weight, even at early progressive stage, may be effective diagnostic protocol for detecting MD-OAD.

Keywords: Muscular dystrophy; Diagnostic protocol; Prognostic biomarkers; Muscle enzymes; Abnormal muscles structure; Anatomical anomalies

\section{Introduction}

Muscular Dystrophy during Osteoarthritic Disorder (MD-OAD) is a collection of disorders attributed by muscle wasting, weakness and degeneration along with chronic arthropathy relating to potential loss of joint cartilage, bone hypertrophy, connective tissue damage, pain symptoms, joint effusion, restricted movement of joints, tenderness and Crepitus in the joints along with abnormal biochemical and anatomical features [1,2]. But muscular dystrophy itself is a chronic progressive disorder because over time healthy muscle fibers are lost and replaced by fibrosis and fat making muscle tissues especially in the thigh region and wasting of calf muscles ensue less able to generate force for the movement of legs. This might be happened due to progressive loss of muscle mass caused by interference with the production of muscle protein called dystrophin, which produce upon

\section{Journal of Orthopedics \& Rheumatology}

\author{
Apurba Ganguly* and Devika Ganguly \\ Department of Research and Development, OPTM Research \\ Institute, India \\ ${ }^{*}$ Address for Correspondence \\ Apurba Ganguly, Founder and Chief Scientist, OPTM Research Institute, \\ Kolkata, India, Tel: +919830389616; E-mail: apurbaganguly15@gmail.com \\ Submission: 04 September, 2018 \\ Accepted: 14 September, 2018 \\ Published: 24 September, 2018 \\ Copyright: (๑ 2018 Ganguly A, et al. This is an open access article \\ distributed under the Creative Commons Attribution License, which \\ permits unrestricted use, distribution, and reproduction in any medium, \\ provided the original work is properly cited.
}

the instructions of the largest gene in the body. This serum protein is located primarily in skeletal muscles used for movement and in the cardiac muscle serves the purpose of normal cardiovascular system. It is known that small amounts of dystrophin are present in nerve cells in the brain and majorly found in muscles [3].

This rod-shaped cytoplasmic dystrophin protein connects the cytoskeletol of a muscle fiber and finally links the various components within the muscle cells to the outer membrane known as sarcolemma. Duchenne Muscular Dystrophy (DMD) and Becker Muscular Dystrophy (BMD) are the most common among the nine types of muscular dystrophies. But the characteristic features of $\mathrm{DMD}$ and $\mathrm{BMD}$ are not the same in MD-OAD except in muscular wasting, muscle weakness, and degeneration of muscle. Besides abnormalities in muscles as mentioned above especially in the thigh and calf muscles, pain and stiffness in the joints, bone erosion, lose of various cartilages, formation of osteophytes, joint effusion, restricted movement of joints, bone marrow oedema, damage of various ligaments, loss of synovitis, particular formation of cysts/ bursitis and deformity of bones thus to form genu varum, may simultaneously occurred in MD-OAD. Thus, it is dependent upon the bone and muscle relationship in the men and women over the age of forty unless otherwise there is accidental damage. It is also closely related to sarcopenia where failure of muscle strength and functional ability are observed due to loss of muscle mass [4-6].

According to Lang, muscles support the bone structure, particularly during the process of growth and development [7]. The close relationship between muscle and bone and this relationship are observed through the mechanostat theory, evaluated firstly to determine gender differences by Frost $[8,9]$. During the attainment of aging, peak bone and muscle strength, both men and women begin to lose both bone and muscle tissues and possibilities of muscles and bone damage widely known as sarcopenia [3-7].

In recent past, researchers have studied muscle damage or muscular dystrophy by evaluating only biochemical markers such as creatine kinase, lactate dehydrogenase, aldolase, myoglobin, troponin, aspartate aminotransferase, and carbonic anhydrase CAIII 
are the most useful serum markers of muscle injury or musculoskeletal ultrasound for soft tissue damage [10,11], infections, muscle and tendon damage, etc [12-16]. or Magnetic Resonance Imaging (MRI) for inflammatory myopathies, knee menisci damage, etc $[17,18]$ or power Doppler sonography for inflammatory musculoskeletal disease, etc $[19,20]$. or muscle pain with index for disability test etc [21]. or radio-diagnosis related to muscle strength and bone deformities or muscle biopsy with special reference to changes in fibre size, changes in fibre-type distribution [22,23], changes in sarcolemmal nuclei, signs of degeneration, cellular reaction, changes in architecture of individual fibres [24]. Ganguly have investigated that important parameters such as levels of calcium, phosphorous, ratio of calcium-phosphorous with high or low value of parathyroid hormone level are identified bone damage diagnosis [25,26].

Interestingly, researchers have investigated Knee-Osteoarthritis (KOA), which occurs due to the damage of leg muscles such as quadriceps femoris, extensor muscle, etc. [22,23]. The bones, muscles and cartilage are the important tissues deformed during KOA as an evidenced by radiographic marker to detect only bone joint space narrowing in the knee [11,18-19,23,24,27-36]. Although, Ganguly has diagnosed abnormal musculo-postural features during KOA [35].

The primary diagnosis of MD-OAD by physical examination with the observation of symptoms such as waddling gait, pain and stiffness in the muscles, difficulty with running and jumping, difficulty in sitting and standing, frequent falls, loss of spine curvature, varus/ valgus deformity, painful joint effusion, warmness, tenderness, crepitus and limited range of motion of the joints. After the physical examination in order to confirm the MD-OAD in multiple joints, the imaging scans such as CT-scan or X-ray or MRI, Electromyography (EMG) for muscle strength, muscle biopsy for abnormal muscle growth with malignancy, genetic tests to confirm heredity problems, heart-monitoring tests such as Electrocardiography (ECG) for abnormalities that may affect heart function, enzyme tests such as CKMM for muscle degeneration and musculoskeletal ultrasounds for neuromuscular disorders, malignancies, infections and hematomas, Computer Tomography (CT) for the evaluation of deepest muscles group disorders. In case of X-rays for multiple joints to examine the condition of bones which has limitations of too much radiation in the body and also restricted to pregnant patients but not identify the quantum of muscle degeneration and skeletal muscle damage and in case of MRI produces images of bones, ligaments, cartilage and synovitis in analyzing shape, volume, and morphological features of skeletal muscle and also can detect inflammatory changes in muscles with some limitations such as certain metal objects implanted in the body viz. pacemakers, prosthetic joints, rods and certain tattoos and also restricted to overweight, very tall and claustrophobic patients. Moreover, MRI is very costly, and it is to be done for multiple joints to indentify the damages during MD-OADs. On the other hand, EMG indicates only skeletal muscle strength not the quantum of damages occur in the joint cartilages and ligaments during MD-OAD, genetic and biopsy tests do not focus on the conditions of the muscles and joints and finally the ultrasound test may be fruitful in some cases but does not exactly pinpoint the disease condition during MD-OAD and at the same time all the above-mentioned tests in various joint are very expensive and not affordable by common people and also time taken.
The present study attempted for the first time to identify that biochemical markers viz. CK-MM and AldoA are the risk factor for MD-OADs. The level of serum CK-MM is considered to be a biochemical marker to detect muscle and connective tissue damage, and elevated level of serum AldoA have been considered a biomarker to identify the skeletal muscle damage [37,38]. Moreover, all the tests can be done easily and are not very expensive. However, a combined approach using all of these biomarkers to detect risk factors for MDOADs from control and experimental groups has not so far been attempted.

There is no medications available to treat MD-OAD permanently except some kinds of Non-Steroidal Anti-Inflammatory Drugs (NSAIDs), corticosteroids for relieving pain, inflammation, stiffness, improvement of quality of life temporarily along with therapies and assistive devices such as range-of-motion and stretching exercises including low-impact aerobic exercises like walking and swimming for joint flexible using knee braces, lumbar and collar belts for stretching and flexing weak muscles and tendons of joints, using mobility aids like canes, walkers, and wheelchair and finally surgery for the correction of spinal curvature and total knee replacement with artificial prosthesis but during Osteoarthritic Disorders with Muscular Dystrophy (MD-OAD) on the multiple joints, surgery is not at all successful having elevated levels of serum CK-MM and AldoA in the blood which lead to further damages of muscle strength and other associated unavoidable complications.

It is known that overall strength of the body for functional ability mainly depends on muscles strength [27-32]. It was observed that there is weakness of the quadriceps muscle during MD-OAD, because quadriceps strength (peak torque generation) is an important evaluation for physical function in men and women [27-32]. The muscle impairment causes pain, inflammation and functional disabilities in the MD-OAD patients [37-44]. But the combination of analyses of biochemical markers and anatomical features along with different indices to diagnosis muscular dystrophy during the progression of OADs are lacking.

The objective of present study was to diagnose the MD-OAD by evaluating the above-mentioned biochemical markers and anatomical features along with different indices in combinations. quickly and in affordable low cost at the early stage of MD-OAD where there is no pain syndrome or discomfort, or deformities observed in the joints much before focusing in the radiological images, EMI, muscle ultrasound tests etc. Therefore, the present study has portent the novelty concepts for diagnosis of MD-OADs into the relevant biomedical and anatomical categories.

\section{Materials and Methods}

\section{Recruitment of participants}

A total of 711 participants, aged 40 to 80 years old who came for the treatment and also the participants who came along with the patients as patient party at OPTM Health Care (P) Ltd. centers in Kolkata, Delhi and Mumbai, India from January 2017 to December 2017 were evaluated in this study. The study protocol was evaluated and approved by the OPTM (Organic Phyto Therapeutic Method) Research Institute Ethics Committee. The institute is registered with the government. An Institutional Review Board-approved consent 
Citation: Ganguly A, Ganguly D. Muscular Dystrophy during Osteoarthritic Disorder: A Novel Diagnostic Protocol Evidenced by Biochemical and Anatomical Parameters. J Orthopedics Rheumatol. 2018; 5(2): 14

ISSN: 2334-2846

Table 1: Demographic data and baseline characteristics of subjects.

\begin{tabular}{|c|c|c|}
\hline Characteristics & Experimental group & Control group \\
\hline No of subjects & 162 & 153 \\
\hline Females & $117(72.22 \%)$ & $108(70.59 \%)$ \\
\hline Age (yrs), [mean (SD)] & $59.89(7.19)$ & $58.96(8.63)$ \\
\hline Height (in m), [mean (SD)] & $1.56(0.72)$ & $1.51(0.88)$ \\
\hline Weight (in kg.), [mean(SD)] & $72.52(4.17)$ & $62.52(4.78)$ \\
\hline BMI $\left(\mathrm{kg} / \mathrm{m}^{2}\right)[$ mean $(\mathrm{SD})]$ & $31.94(3.31)$ & $27.42(3.38)$ \\
\hline Period of suffering (yrs), [mean(SD)] & $7.89(1.90)$ & - \\
\hline \multicolumn{3}{|c|}{ Indian ethnic group (\%) } \\
\hline Bengali & $51(31.48)$ & $49(32.03)$ \\
\hline Gujrati & $14(8.64)$ & $13(8.50)$ \\
\hline Marwaree & $11(6.79)$ & $12(7.84)$ \\
\hline Marathi & $19(11.73)$ & $18(11.76)$ \\
\hline Punjabi & $18(11.11)$ & $17(11.11)$ \\
\hline Shindhi & $19(11.74)$ & $16(10.46)$ \\
\hline North East India & $13(8.02)$ & $12(7.84)$ \\
\hline \multicolumn{3}{|c|}{ Food habit (\%) } \\
\hline Vegetarian & $98(60.49)$ & $88(57.52)$ \\
\hline Non - vegetarian & $64(39.51)$ & $65(42.48)$ \\
\hline \multicolumn{3}{|c|}{ Other habits (\%) } \\
\hline Drinking excessive tea and coffee & $59(36.42)$ & $55(35.95)$ \\
\hline Smoking & $48(29.63)$ & $46(30.06)$ \\
\hline Drinking Alcohol & $41(25.31)$ & $39(25.49)$ \\
\hline Chewing tobacco & $14(8.64)$ & $13(8.50)$ \\
\hline \multicolumn{3}{|c|}{ Work status (\%) } \\
\hline Employed fulltime & $58(35.80)$ & 55 (35.95) \\
\hline Housewife / Homemaker & $52(32.10)$ & $49(32.03)$ \\
\hline Retired & $25(15.43)$ & $21(13.32)$ \\
\hline Self employed & $16(9.88)$ & $18(11.76)$ \\
\hline \multicolumn{3}{|c|}{ Multiple complaints (\%) } \\
\hline Constipation & $68(41.97)$ & $80(52.29)$ \\
\hline Acidity \& reflux & $72(44.44)$ & $45(29.41)$ \\
\hline Insomnia & $66(40.74)$ & $62(40.52)$ \\
\hline Varicose vein & 34 (20.99) & $32(20.91)$ \\
\hline Urinary incontinence & $43(26.54)$ & $17(11.11)$ \\
\hline Crepitus during knee flexion & $32(19.75)$ & - \\
\hline Morning stiffness (<30 minute) & $37(22.84)$ & - \\
\hline \multicolumn{3}{|c|}{ Measures taken to diminish pain (\%) } \\
\hline Using knee caps & $68(41.98)$ & - \\
\hline Using a lumbar belt & $48(29.63)$ & - \\
\hline Using a collar belt & $35(21.60)$ & - \\
\hline Using a stick/walker & $34(20.99)$ & - \\
\hline \multicolumn{3}{|c|}{ Katz index of independence in ADL (\%) } \\
\hline Bathing & $111(68.52)$ & $(100)$ \\
\hline Dressing & $107(66.05)$ & $(100)$ \\
\hline Toileting & $98(60.49)$ & $(100)$ \\
\hline Transferring & $110(67.90)$ & $(100)$ \\
\hline Continence & $119(73.46)$ & $(100)$ \\
\hline Feeding & $132(81.48)$ & $(100)$ \\
\hline
\end{tabular}


Citation: Ganguly A, Ganguly D. Muscular Dystrophy during Osteoarthritic Disorder: A Novel Diagnostic Protocol Evidenced by Biochemical and Anatomical Parameters. J Orthopedics Rheumatol. 2018; 5(2): 14

Table 2A: Statistical analysis of biochemical parameters of 162 experimental and 153 control subjects.

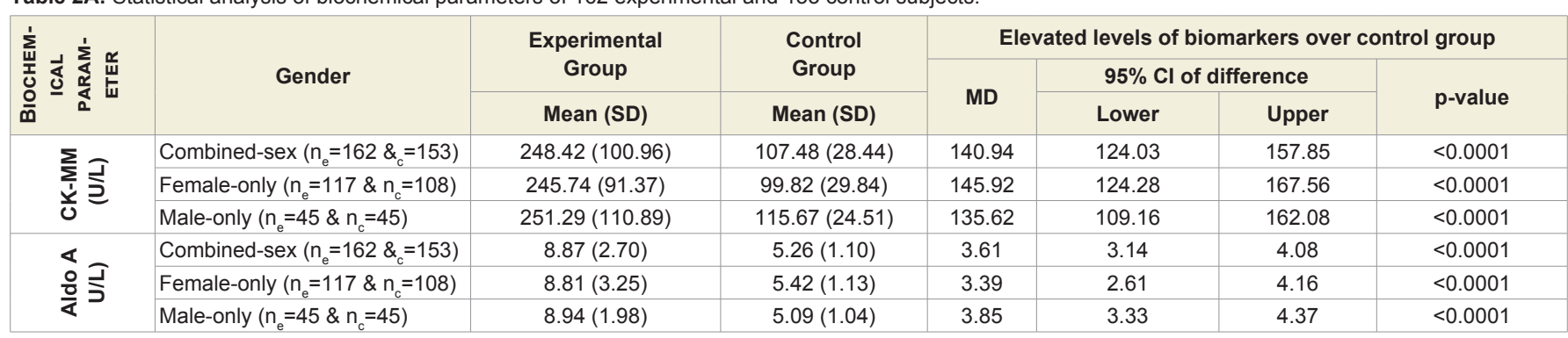

Table 2B: Analysis of ratio of CK-MM and Aldo A of 162 experimental patients and 153 control subjects.

\begin{tabular}{|c|c|c|c|c|c|c|}
\hline & \multirow{3}{*}{$\begin{array}{c}\text { Experimental Group } \\
\text { CK-MM : Aldo A } \\
\text { Mean (SD) }\end{array}$} & \multirow{3}{*}{$\begin{array}{c}\text { Control Group } \\
\text { CK-MM : Aldo A } \\
\text { Mean (SD) }\end{array}$} & \multicolumn{4}{|c|}{ Improvement after treatment } \\
\hline & & & & & \multicolumn{2}{|c|}{$95 \% \mathrm{Cl}$} \\
\hline & & & & & Lower & Upper \\
\hline Combined-sex $\left(n_{e}=162 \& n_{c}=153\right)$ & $29.71(13.07)$ & $21.46(7.79)$ & $<0.0001$ & 8.25 & 5.8 & 10.7 \\
\hline Female-only $\left(n_{e}=117 \& n_{c}=108\right)$ & $30.82(11.90)$ & $19.22(7.21)$ & $<0.0001$ & 11.6 & 8.47 & 14.73 \\
\hline Male-only $\left(n_{e}=45 \& n_{c}=45\right)$ & $29.37(14.29)$ & $23.86(7.32)$ & $<0.0001$ & 5.51 & 1.77 & 9.25 \\
\hline
\end{tabular}

Table 3: Analysis of Pearson's correlation coefficients between two biomarkers.

\begin{tabular}{|c|c|c|c|c|c|c|c|c|c|c|}
\hline \multirow{2}{*}{ Gender } & \multicolumn{2}{|c|}{$\begin{array}{l}\text { CK-MM }{ }^{\operatorname{Exp}} \\
\text { and } \\
\text { CK-MM }\end{array}$} & \multicolumn{2}{|c|}{$\begin{array}{l}\text { Aldo }^{\operatorname{Exp}} \\
\text { and } \\
\text { Aldo }^{\text {Con }}\end{array}$} & \multicolumn{2}{|c|}{$\begin{array}{l}\text { CK-MM } M^{\operatorname{Exp}} \\
\text { and } \\
\text { AldoA }^{\operatorname{Exp}}\end{array}$} & \multicolumn{2}{|c|}{$\begin{array}{l}\text { CK-MM }^{\text {Con }} \\
\text { and } \\
\text { AldoA }^{\text {con }}\end{array}$} & \multicolumn{2}{|c|}{$\begin{array}{l}\text { CK-MM }{ }^{\text {Exp }} \\
\text { and } \\
\text { Aldo }^{\text {Con }}\end{array}$} \\
\hline & R-value & p-value & R-value & p-value & R-value & p-value & R-value & $p$-value & R-value & p-value \\
\hline $\begin{array}{l}\text { Combined-sex }\left(n_{e}=162 \&\right. \\
\left.n_{c}=153\right)\end{array}$ & 0.494 & 0.000 & 0.241 & 0.000 & 0.320 & 0.000 & 0.118 & 0.057 & 0.253 & 0.000 \\
\hline $\begin{array}{l}\text { Female-only } \\
\left(n_{e}=117 \& n_{c}=108\right)\end{array}$ & 0.505 & 0.000 & 0.414 & 0.000 & 0.325 & 0.005 & 0.183 & 0.016 & 0.285 & 0.000 \\
\hline $\begin{array}{l}\text { Male-only } \\
\left(n_{e}=45 \& n_{c}=45\right)\end{array}$ & 0.228 & 0.034 & -0.103 & 0.041 & 0.158 & 0.043 & 0.008 & 0.942 & 0.247 & 0.021 \\
\hline
\end{tabular}

form for the physical examinations, blood sample collections, electromyography, muscle biopsy and heart-monitoring tests, skeletal muscle ultrasound and radiological images (CT-scan or X-ray or MRI) required for the study was signed by all patients in the first phase of the screening procedure.

\section{Exclusion criteria}

Three hundred and ninety three of 711 participants were excluded for having another pathological condition that could explain the existing symptoms, such as rheumatic diseases, osteochondritis diseases, inter articular fractures, congenital dysplasia, radicular syndrome, joint symptoms caused by malignant tumors, baker's cyst, perthes disease, plico syndrome, dermatomyositis and polymyositis diseases, iliopectineal or trochanteric bursitis, bone and joint infectious diseases and ischemic bone necrosis. The following additional exclusion criteria were considered: patients with multiple drug dependence; a history of cancer, including carcinomatosis and granulocytic leukemia; patients with cuts, wounds, or any type of chronic skin disease; a history of severe neurological diseases; a history of chronic liver, kidney and heart diseases; and patients who did not agree to a physical evaluation and/or attend weekly follow-up visits.

\section{Study design}

After evaluating the exclusion criteria, 162 of the remaining 315 subjects with significant pain syndromes, discomfort, imbalanced quality of life, impaired joint and limb functions due to muscle wasting, weakness and degeneration in multiple regions of the body, as evidenced by elevated levels of serum CK-MM and AldoA, electromyography, muscle ultrasound, muscle biopsy and heartmonitoring tests and radiological images (CT-scan or X-ray or MRI) were considered as experimental subjects and termed as 'subjects with MD-OAD' The remaining 153 subjects who had no complain of pain, discomfort in quality of life and no abnormalities observed in muscles, in joint and limb functions in any region, jointly or singly, according to the analyses of serum CK-MM and AldoA, electromyography, muscle biopsy, muscle ultrasound, heartmonitoring tests and radiological images were considered as healthy control subjects and termed as 'subjects without MD-OAD'. The baseline demographic characteristics of all patients are presented in Table 1. Co-morbidities were also assessed using the Charlson comorbidity index and methods described by Katz et al. and Singh et al. $[45,46]$. 
Citation: Ganguly A, Ganguly D. Muscular Dystrophy during Osteoarthritic Disorder: A Novel Diagnostic Protocol Evidenced by Biochemical and Anatomical Parameters. J Orthopedics Rheumatol. 2018; 5(2): 14.

Table 4A: Statistical analysis of rang of motions of 162 experimental subjects and 153 control subjects.

\begin{tabular}{|c|c|c|c|c|c|c|c|c|c|c|c|}
\hline \multirow{3}{*}{ 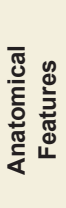 } & \multirow{3}{*}{ Gender } & \multicolumn{3}{|c|}{ Experiment Group } & \multicolumn{3}{|c|}{ Control Group } & \multicolumn{4}{|c|}{$\begin{array}{c}\text { Increased/decreased anatomical parameters over } \\
\text { Control group }\end{array}$} \\
\hline & & \multirow{2}{*}{\begin{tabular}{|c|} 
Rt. leg \\
Mean (SD)
\end{tabular}} & \multirow{2}{*}{\begin{tabular}{c|} 
Lt. leg \\
Mean (SD)
\end{tabular}} & \multirow{2}{*}{\begin{tabular}{|c|} 
DBL \\
Mean (SD)
\end{tabular}} & \multirow{2}{*}{\begin{tabular}{|c|} 
Rt. leg \\
Mean (SD)
\end{tabular}} & \multirow{2}{*}{\begin{tabular}{c|} 
Lt. leg \\
Mean (SD)
\end{tabular}} & \multirow{2}{*}{$\begin{array}{c}\text { DBL } \\
\text { Mean (SD) }\end{array}$} & \multicolumn{2}{|c|}{ Right Leg } & \multicolumn{2}{|c|}{ Left Leg } \\
\hline & & & & & & & & $\begin{array}{c}\mathrm{MD} \\
(95 \% \mathrm{Cl})\end{array}$ & $p$-value & $\begin{array}{c}\mathrm{MD} \\
(95 \% \mathrm{Cl})\end{array}$ & $p$-value \\
\hline \multirow{3}{*}{ 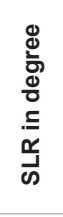 } & $\begin{array}{l}\text { Combined-sex } \\
\left(n_{e}=162 \& n_{c}=153\right)\end{array}$ & $\begin{array}{c}53.01 \\
(10.07)\end{array}$ & $\begin{array}{l}53.94 \\
(9.52)\end{array}$ & $\begin{array}{c}2.79 \\
(1.70)\end{array}$ & $\begin{array}{l}75.87 \\
(3.78)\end{array}$ & $\begin{array}{l}75.87 \\
(3.78)\end{array}$ & $\begin{array}{c}0 \\
(0)\end{array}$ & $\begin{array}{c}-22.56 \\
(-24.26,-20.86)\end{array}$ & $<0.001$ & $\begin{array}{c}-21.63 \\
(-23.25,-20.01) \\
\end{array}$ & $<0.001$ \\
\hline & $\begin{array}{l}\text { Female-only } \\
\left(n_{e}=117 \& n_{c}=108\right)\end{array}$ & $\begin{array}{c}53.11 \\
(10.52)\end{array}$ & $\begin{array}{l}54.12 \\
(9.84)\end{array}$ & $\begin{array}{c}2.84 \\
(1.75)\end{array}$ & $\begin{array}{l}75.59 \\
(3.73)\end{array}$ & $\begin{array}{l}75.59 \\
(3.73)\end{array}$ & $\begin{array}{c}0 \\
(0)\end{array}$ & $\begin{array}{c}-22.48 \\
(-24.59,-20.37)\end{array}$ & $<0.001$ & $\begin{array}{c}-22.47 \\
(-24.46,-20.48)\end{array}$ & $<0.001$ \\
\hline & $\begin{array}{l}\text { Male-only } \\
\left(n_{e}=45 \& n_{c}=45\right)\end{array}$ & $\begin{array}{l}53.02 \\
(8.92)\end{array}$ & $\begin{array}{l}53.22 \\
(8.69)\end{array}$ & $\begin{array}{c}2.69 \\
(1.56)\end{array}$ & $\begin{array}{l}75.29 \\
(3.75)\end{array}$ & $\begin{array}{l}75.29 \\
(3.75)\end{array}$ & $\begin{array}{c}0 \\
(0)\end{array}$ & $\begin{array}{c}-22.27 \\
(-25.14,-19.40)\end{array}$ & $<0.001$ & $\begin{array}{c}-22.07 \\
(-24.87,19.82)\end{array}$ & $<0.001$ \\
\hline \multirow{3}{*}{ 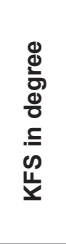 } & $\begin{array}{l}\text { Combined-sex } \\
\left(n_{e}=162 \& n_{c}=153\right)\end{array}$ & $\begin{array}{l}112.68 \\
(6.90)\end{array}$ & $\begin{array}{l}112.84 \\
(6.51)\end{array}$ & $\begin{array}{c}3.08 \\
(2.06)\end{array}$ & $\begin{array}{l}143.06 \\
(1.85)\end{array}$ & $\begin{array}{l}143.06 \\
(1.85)\end{array}$ & $\begin{array}{l}0 \\
0\end{array}$ & $\begin{array}{c}-30.38 \\
(-31.56,-29.24) \\
\end{array}$ & $<0.001$ & $\begin{array}{c}-30.22 \\
(-31.29,-29.14)\end{array}$ & $<0.001$ \\
\hline & $\begin{array}{l}\text { Female-only } \\
\left(n_{e}=117 \& n_{c}=108\right)\end{array}$ & $\begin{array}{l}112.54 \\
(7.00)\end{array}$ & $\begin{array}{l}112.76 \\
(6.67)\end{array}$ & $\begin{array}{c}3.18 \\
(2.17)\end{array}$ & $\begin{array}{l}143.10 \\
(1.84)\end{array}$ & $\begin{array}{l}143.10 \\
(1.84)\end{array}$ & $\begin{array}{c}0 \\
(0)\end{array}$ & $\begin{array}{c}-30.56 \\
(-31.93,-29.14)\end{array}$ & $<0.001$ & $\begin{array}{c}-30.34 \\
(-31.65,-29.03)\end{array}$ & $<0.001$ \\
\hline & $\begin{array}{l}\text { Male-only } \\
\left(n_{e}=45 \& n_{c}=45\right)\end{array}$ & $\begin{array}{l}112.69 \\
(6.71)\end{array}$ & $\begin{array}{l}113.16 \\
(6.15)\end{array}$ & $\begin{array}{c}2.82 \\
(1.72)\end{array}$ & $\begin{array}{l}143.16 \\
(1.88)\end{array}$ & $\begin{array}{l}143.16 \\
(1.88)\end{array}$ & $\begin{array}{c}0 \\
(0)\end{array}$ & $\begin{array}{c}-30.47 \\
(-32.53,-28.41)\end{array}$ & $<0.001$ & $\begin{array}{c}-30.00 \\
(-31.90,28.09)\end{array}$ & $<0.001$ \\
\hline \multirow{3}{*}{ 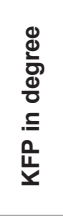 } & $\begin{array}{l}\text { Combined-sex } \\
\left(n_{e}=162 \& n_{c}=153\right)\end{array}$ & $\begin{array}{l}108.49 \\
(5.92)\end{array}$ & $\begin{array}{l}108.96 \\
(5.50)\end{array}$ & $\begin{array}{c}2.63 \\
(1.77) \\
\end{array}$ & $\begin{array}{l}140.74 \\
(1.83)\end{array}$ & $\begin{array}{l}140.74 \\
(1.83)\end{array}$ & $\begin{array}{c}0 \\
(0)\end{array}$ & $\begin{array}{c}-32.25 \\
(-33.23,-31.67)\end{array}$ & $<0.001$ & $\begin{array}{c}-31.78 \\
(-32.70,30.86)\end{array}$ & $<0.001$ \\
\hline & $\begin{array}{l}\text { Female-only } \\
\left(n_{e}=117 \& n_{c}=108\right)\end{array}$ & $\begin{array}{l}108.40 \\
(5.87)\end{array}$ & $\begin{array}{l}108.87 \\
(5.51)\end{array}$ & $\begin{array}{c}2.68 \\
(1.88)\end{array}$ & $\begin{array}{l}140.56 \\
(1.84)\end{array}$ & $\begin{array}{l}140.56 \\
(1.84)\end{array}$ & $\begin{array}{c}0 \\
(0)\end{array}$ & $\begin{array}{c}-32.16 \\
(-33.32,-31.00)\end{array}$ & $<0.001$ & $\begin{array}{c}-31.69 \\
(-32.79,30.59)\end{array}$ & $<0.001$ \\
\hline & $\begin{array}{l}\text { Male-only } \\
\left(n_{e}=45 \& n_{c}=45\right)\end{array}$ & $\begin{array}{c}108.62 \\
(6.11)\end{array}$ & $\begin{array}{l}109.42 \\
(5.50)\end{array}$ & $\begin{array}{c}2.44 \\
(1.42)\end{array}$ & $\begin{array}{c}141.16 \\
(1.74)\end{array}$ & $\begin{array}{l}141.16 \\
(1.74)\end{array}$ & $\begin{array}{c}0 \\
(0)\end{array}$ & $\begin{array}{c}-32.54 \\
(-34.42,-30.66)\end{array}$ & $<0.001$ & $\begin{array}{c}-31.74 \\
(-33.45,30.03)\end{array}$ & $<0.001$ \\
\hline \multirow{3}{*}{ 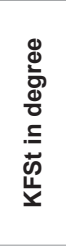 } & $\begin{array}{l}\text { Combined-sex } \\
\left(n_{e}=162 \&\right. \\
\left.n_{c}=153\right)\end{array}$ & $\begin{array}{c}103.24 \\
(9.57)\end{array}$ & $\begin{array}{l}103.20 \\
(9.45)\end{array}$ & $\begin{array}{c}2.47 \\
(1.72)\end{array}$ & $\begin{array}{l}140.24 \\
(1.84)\end{array}$ & $\begin{array}{c}140.24 \\
(1.84)\end{array}$ & $\begin{array}{c}0 \\
(0)\end{array}$ & $\begin{array}{c}-37.00 \\
(-38.56,-35.45)\end{array}$ & $<0.001$ & $\begin{array}{c}-37.04 \\
(-38.57,-35.51)\end{array}$ & $<0.001$ \\
\hline & $\begin{array}{l}\text { Female-only } \\
\left(n_{e}=117 \& n_{c}=108\right)\end{array}$ & $\begin{array}{l}102.87 \\
(9.97)\end{array}$ & $\begin{array}{l}102.68 \\
(9.93)\end{array}$ & $\begin{array}{c}2.53 \\
(1.81)\end{array}$ & $\begin{array}{l}140.47 \\
(1.81)\end{array}$ & $\begin{array}{c}140.47 \\
(1.81\end{array}$ & $\begin{array}{c}0 \\
(0)\end{array}$ & $\begin{array}{c}-37.60 \\
(-39.52,-35.68)\end{array}$ & $<0.001$ & $\begin{array}{c}-37.79 \\
(-39.70,-35.88)\end{array}$ & $<0.001$ \\
\hline & $\begin{array}{l}\text { Male-only } \\
\left(n_{e}=45 \& n_{c}=45\right)\end{array}$ & $\begin{array}{l}104.11 \\
(8.46)\end{array}$ & $\begin{array}{l}104.76 \\
(7.97)\end{array}$ & $\begin{array}{c}2.24 \\
(1.48) \\
\end{array}$ & $\begin{array}{l}139.40 \\
(1.72)\end{array}$ & $\begin{array}{l}139.40 \\
(1.72)\end{array}$ & $\begin{array}{c}0 \\
(0)\end{array}$ & $\begin{array}{c}-37.29 \\
(-37.85,-32.73)\end{array}$ & $<0.001$ & $\begin{array}{c}-34.64 \\
(-37.05,-32.22)\end{array}$ & $<0.001$ \\
\hline \multirow{2}{*}{ 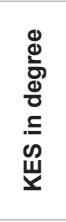 } & $\begin{array}{l}\text { Female-only } \\
\left(n_{e}=117 \& n_{c}=108\right)\end{array}$ & $\begin{array}{l}17.69 \\
(2.20)\end{array}$ & $\begin{array}{l}17.48 \\
(1.76)\end{array}$ & $\begin{array}{c}1.38 \\
(1.66)\end{array}$ & $\begin{array}{c}8.94 \\
(0.69)\end{array}$ & $\begin{array}{c}8.94 \\
(0.69\end{array}$ & $\begin{array}{c}0 \\
(0)\end{array}$ & $\begin{array}{c}8.75 \\
(8.13,9.18)\end{array}$ & $<0.001$ & $\begin{array}{c}8.54 \\
(8.18,8.90)\end{array}$ & $<0.001$ \\
\hline & $\begin{array}{l}\text { Male-only } \\
\left(n_{e}=45 \& n_{c}=45\right)\end{array}$ & $\begin{array}{l}17.22 \\
(1.18) \\
\end{array}$ & $\begin{array}{l}17.53 \\
(1.12)\end{array}$ & $\begin{array}{c}1.07 \\
(0.25)\end{array}$ & $\begin{array}{c}9.41 \\
(0.83)\end{array}$ & $\begin{array}{c}9.41 \\
(0.83)\end{array}$ & $\begin{array}{l}0 \\
0)\end{array}$ & $\begin{array}{c}7.81 \\
(7.38,8.24)\end{array}$ & $<0.001$ & $\begin{array}{c}8.12 \\
(7.71,8.50)\end{array}$ & $<0.001$ \\
\hline \multirow{3}{*}{ 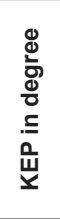 } & $\begin{array}{l}\text { Combined-sex } \\
\left(n_{e}=162 \& n_{c}=153\right)\end{array}$ & $\begin{array}{l}17.29 \\
(1.69)\end{array}$ & $\begin{array}{l}17.01 \\
(1.74)\end{array}$ & $\begin{array}{c}1.24 \\
(0.93)\end{array}$ & $\begin{array}{l}10.05 \\
(0.58)\end{array}$ & $\begin{array}{l}10.05 \\
(0.58)\end{array}$ & $\begin{array}{c}0 \\
(0)\end{array}$ & $\begin{array}{c}7.24 \\
(6.96,7.52)\end{array}$ & $<0.001$ & $\begin{array}{c}6.93 \\
(6.67,7.26)\end{array}$ & $<0.001$ \\
\hline & $\begin{array}{l}\text { Female-only } \\
\left(n_{e}=117 \& n_{c}=108\right)\end{array}$ & $\begin{array}{l}17.33 \\
(1.83)\end{array}$ & $\begin{array}{l}16.97 \\
(1.85)\end{array}$ & $\begin{array}{c}1.27 \\
(1.08) \\
\end{array}$ & $\begin{array}{l}10.00 \\
(0.63)\end{array}$ & $\begin{array}{l}10.00 \\
(0.63)\end{array}$ & $\begin{array}{c}0 \\
(0)\end{array}$ & $\begin{array}{c}7.33 \\
(6.94,7.69)\end{array}$ & $<0.001$ & $\begin{array}{c}6.97 \\
(6.60,7.39)\end{array}$ & $<0.001$ \\
\hline & $\begin{array}{l}\text { Male-only } \\
\left(n_{e}=45 \& n_{c}=45\right)\end{array}$ & $\begin{array}{l}17.07 \\
(1.25)\end{array}$ & $\begin{array}{l}16.93 \\
(1.42)\end{array}$ & $\begin{array}{c}1.07 \\
(0.25)\end{array}$ & $\begin{array}{l}10.20 \\
(0.40)\end{array}$ & $\begin{array}{l}10.20 \\
(0.40)\end{array}$ & $\begin{array}{c}0 \\
(0)\end{array}$ & $\begin{array}{c}6.87 \\
(6.48,7.26)\end{array}$ & $<0.001$ & $\begin{array}{c}6.73 \\
(6.29,7.17)\end{array}$ & $<0.001$ \\
\hline \multirow{3}{*}{ 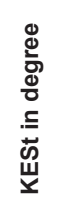 } & $\begin{array}{l}\text { Combined-sex } \\
\left(n_{e}=162 \& n_{c}=153\right)\end{array}$ & $\begin{array}{l}16.84 \\
(2.15)\end{array}$ & $\begin{array}{l}16.49 \\
(2.07)\end{array}$ & $\begin{array}{c}1.34 \\
(1.47)\end{array}$ & $\begin{array}{l}10.62 \\
(0.46)\end{array}$ & $\begin{array}{l}10.62 \\
(0.46)\end{array}$ & $\begin{array}{c}0 \\
(0)\end{array}$ & $\begin{array}{c}6.22 \\
(6.87,6.56)\end{array}$ & $<0.001$ & $\begin{array}{c}6.87 \\
(5.53,6.24)\end{array}$ & $<0.001$ \\
\hline & $\begin{array}{l}\text { Female-only } \\
\left(n_{e}=117 \& n_{c}=108\right)\end{array}$ & $\begin{array}{l}16.85 \\
(2.36)\end{array}$ & $\begin{array}{l}16.38 \\
(2.14)\end{array}$ & $\begin{array}{c}1.40 \\
(1.66)\end{array}$ & $\begin{array}{l}10.72 \\
(0.39)\end{array}$ & $\begin{array}{l}10.72 \\
(0.39)\end{array}$ & $\begin{array}{c}0 \\
(0)\end{array}$ & $\begin{array}{c}6.13 \\
(6.68,6.58)\end{array}$ & $<0.001$ & $\begin{array}{c}5.66 \\
(5.25,6.07\end{array}$ & $<0.001$ \\
\hline & $\begin{array}{l}\text { Male-only } \\
\left(n_{e}=45 \& n_{c}=45\right)\end{array}$ & $\begin{array}{l}16.60 \\
\{1.48)\end{array}$ & $\begin{array}{l}16.44 \\
(1.87)\end{array}$ & $\begin{array}{l}1.18 \\
(0.78)\end{array}$ & $\begin{array}{l}10.42 \\
(0.54)\end{array}$ & $\begin{array}{l}10.42 \\
(0.54)\end{array}$ & $\begin{array}{c}0 \\
(0)\end{array}$ & $\begin{array}{c}6.18 \\
(5.71,6.650)\end{array}$ & $<0.001$ & $\begin{array}{c}6.02 \\
(5.44,6.60)\end{array}$ & $<0.001$ \\
\hline
\end{tabular}

\section{Evaluation of specific biochemical parameters in blood}

A $5 \mathrm{ml}$ blood sample was collected in a plain vial from each cohort of experimental and control groups. Blood samples were then centrifuged at $1000 \times \mathrm{g}$ for $10 \mathrm{~min}$ at $4{ }^{\circ} \mathrm{C}$ to obtain serum. Finally, the serum was used to analyse CK-MM and AldoA levels for each cohort of two groups. The biomarkers were rigorously analysed. Each test for each subject has been rechecked by the BS-240 Mindray fully automated biochemistry analyser before reporting the final test results of both the groups.
CK-MM (U/L) levels were quantitatively assessed using a Creatine Kinase-MM kit (CK-MM/CPK-MM/CK-3) and an immunoassay (Aalto Scientific, Limited, USA). The kit was developed based on the methods reported by Cabaniss [47]. AldoA levels (U/L) were quantified using an ALDOLASE (ALS) RX MONZA AD 189 kit (Randox Laboratories Ltd, Antrim, UK) based on a photometric assay at a wavelength of $340 \mathrm{~nm}$. The kit was developed according to the method reported by Feissli [48]. The serum of each subject of both the groups was tested for CK-MM, and AldoA levels separately. All blood tests were conducted under the supervision of the Chief 
Citation: Ganguly A, Ganguly D. Muscular Dystrophy during Osteoarthritic Disorder: A Novel Diagnostic Protocol Evidenced by Biochemical and Anatomical Parameters J Orthopedics Rheumatol. 2018; 5(2): 14

ISSN: $2334-2846$

Table 4B: Statistical Analysis of KGB, DAP, DBP, DCM \& DTM of 162 Experimental Subjects and 171 Control Subjects

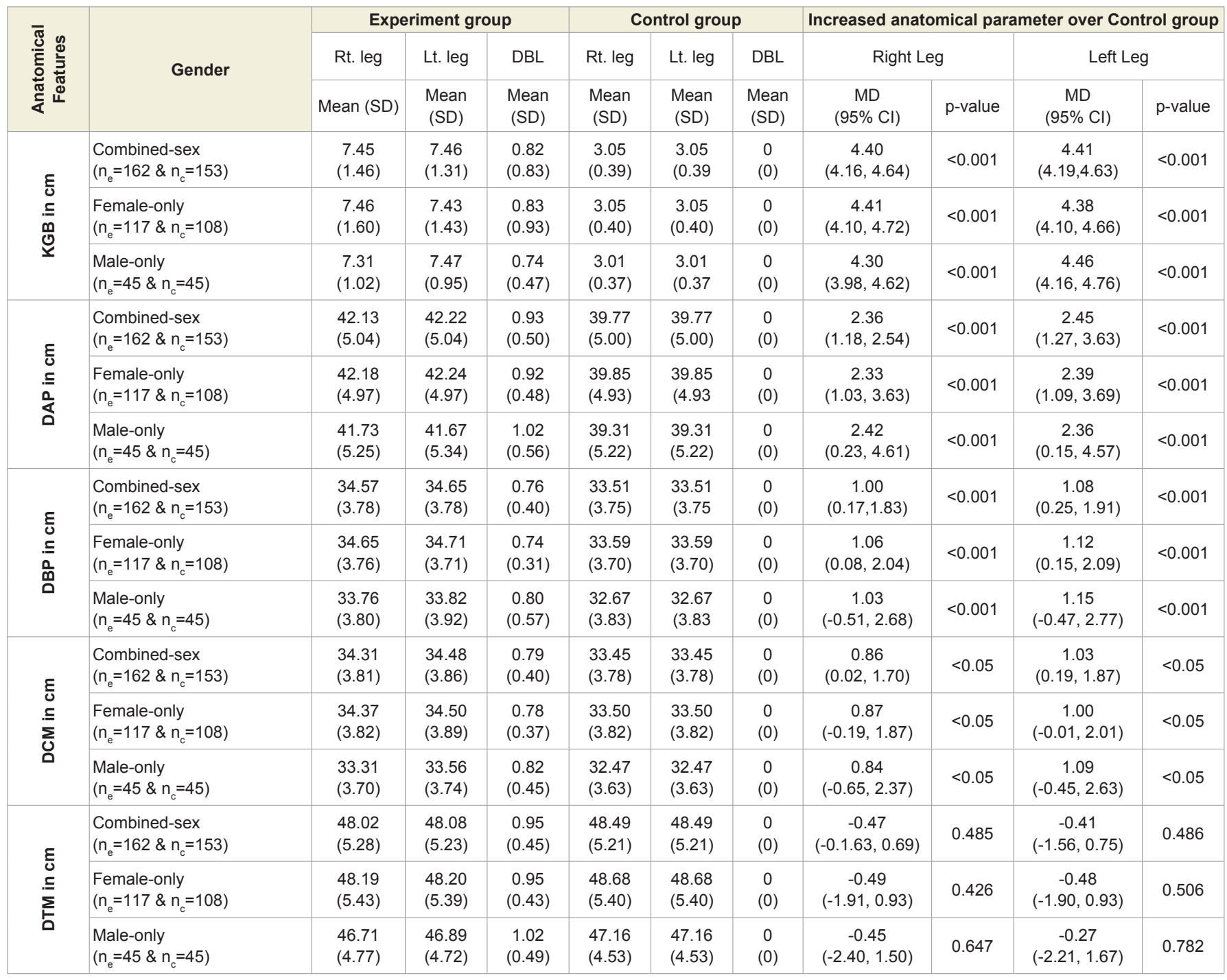

biochemist in the Galaxy Medical Centre, an ISO 9001: 2015 certified lab with Registration No. L/004-(05)-15/0129 under the W.B Clinical Establishment Act, 1950.

\section{Evaluation of Pearson's correlation coefficients between two} biomarkers

To determine the predictive values of the two biomarkers in patients with MD-OADs, the Pearson's correlation coefficients between two biochemical markers such as CK-MM for experimental cohorts with MD-OADs (CK-MM ${ }^{\mathrm{Exp}}$ ) and CK-MM for non- MDOAD control cohorts (CK-MM ${ }^{\mathrm{Con}}$ ), AldoA for experimental cohorts with MD-OADs (AldoA ${ }^{\text {Exp }}$ ) and AldoA for non- MD-OAD control cohorts (Aldo ${ }^{\text {Con }}$, the ratio of CK-MM and AldoA for experimental cohorts with MD-OAD (CK-MM ${ }^{\text {Exp }}$ : Aldo ${ }^{\text {Exp }}$ ) and CK-MM and Aldo A for non- MD-OAD control cohorts (CK-MM ${ }^{\mathrm{Con}}$ : AldoA $\left.{ }^{\mathrm{Con}}\right)$ along with their respective $\mathrm{p}$-values were separately evaluated.

\section{Evaluation of anatomical parameters}

All participants were examined at baseline for signs of musculoskeletal and nervous system damage typically associated with and without MD-OAD in the following locations: patella, tarsal joint, abductor hallucis, abductor digitorium, gastrocnemius, vastus medialis, rectus femoris, vastus lateralis, gracilis,psoas/illiacus, tensor fascia lata, hamstring and popliteus muscles.

Additionally, the following parameters were checked and measured for both legs of all patients of both the groups: the gap at the knee between the point of the short head of the biceps femoris at the lateral knee and the surface of the bed while supine (KGB), diameter of the muscles connected with knee joint $4 \mathrm{~cm}$ above the patella (DAP), diameter of the muscles connected with knee joint $4 \mathrm{~cm}$ below the patella (DBP), diameter of the muscles of thigh (DTM), diameter of the muscles of calf (DCM), knee flexion while supine (KFS), knee flexion while prone (KFP), knee flexion while standing (KFSt), knee extension while supine (KES), knee extension while prone (KEP) and knee extension while standing (KESt).

In the present study, a meter scale was used to measure KGB. DTM, DAP, DBP and DCM measurements were performed using a meter tape and a goniometer was used for all flexion and extension measurements in accordance with the American Academy of 
Orthopedic Surgeons (AAOS) [49]. Evaluations of measurements of difference between legs were also performed to identify muscular wasting and bone deformities in both study groups.

\section{Evaluation of the Katz ADL index for muscular dystrophy}

The Katz index of independence in Activities of Daily Living commonly known as Katz ADL index [50]. This scale is the most appropriate instruments to assess functional status as a measurement of patient's ability to perform activities of daily living independently. The functional status varies from patient to patient due to development of sarcopenia in which reduction of muscle mass was observed followed by failure of muscle strength and functional ability. According to the scale, there are six functions such as bathing, dressing, toileting, transferring, continence and feeding ranked as adequacy of performance. In the scale the scoring system has been marked as 6 for full function, 4 for moderate impairment and 2 or less for severe functional impairment. The functional status in activities of daily living was evaluated for experimental subjects and control subjects in overall and separately by gender. Their mean, SD and p-values for both overall and separately by gender were also graphically evaluated.

\section{Evaluation of Karnofsky Performance Status (KPS) scores}

Karnofsky Performance Status (KPS) score is used to determine a cohort's prognosis to carry out daily activities. A higher score indicates the cohorts are better able to carry out daily activities and its range from 0 to 100 [51]. The KPS is evaluated separately for each cohort of experimental and control groups. The percentage of decreased ability to carry out daily activities was evaluated for each experimental subject compared to the subject of control group separately. Their mean, SD and p-values for overall and separately by gender were graphically evaluated.

\section{Evaluation of Knee-injury Osteoarthritis Outcomes Scale (KOOS) including Quality of life}

The Knee-injury Osteoarthritis Outcomes Scale (KOOS) developed by Ewa Roos and co-authors in the 1990s to assess the patient's opinion about their knee and associated problems including quality of life as an instrument [52]. A Likert scale is used and all items have five possible answer options scored from 0 to 4 and each of the five scores is calculated as the sum of the items included. It holds 42 items in five separately scored subscales experienced during last week such as Pain (0-36); Other symptoms (0-28); function, daily living or Activities of Daily Living (ADL) (0-68); Function, Sport and recreation activities (0-20); and Quality of Life (QOL) (0-16). Scores are transformed to a 0-100 scale with zero representing extreme knee problems and 100 representing no knee problems as common in orthopedic assessment scales and generic measures. Scores between 0 and 100 represent the percentage of total possible score achieved. All the scoring data under KOOS were evaluated separately for each cohort of experimental and control groups. Their mean, SD and p-values for both overall and separately by gender were also graphically evaluated.

\section{Evaluation of Body Mass Index (BMI)}

Body weight (kilogram) was measured without shoes or heavy clothing using an electronic scale. Height (meter) was measured without shoes using a wall-mounted Stadiometer [53]. Body Mass Index (BMI) measured in kg. $\mathrm{m}^{-2}$, was calculated for all the cohorts both experimental and control groups based on their measured weights and heights. BMI was categorized as $>30.0 \mathrm{~kg} / \mathrm{m}^{2}$ (obese), 25.0 to $29.9 \mathrm{~kg} / \mathrm{m}^{2}$ (overweight), 18.5 to $24.9 \mathrm{~kg} / \mathrm{m}^{2}$ (normal weight) and $<18.5 \mathrm{~kg} / \mathrm{m}^{2}$ (underweight). The overall and sex-specific means and standard deviations of each subject of both the groups were evaluated. The percentage of increased BMI of all experimental subjects was evaluated compared with the subjects of control group. Their mean, $\mathrm{SD}$ and $\mathrm{p}$-values for overall and separately by gender were also graphically evaluated.

\section{External study reviewers}

All results and data of experimental subjects suffering with MD$\mathrm{OAD}$ and control group, subjects without MD-OAD were evaluated by an external reviewing panel, not in contract with the registry cohorts.

\section{Data collection and statistical analyses}

Data were summarized using descriptive statistics for continuous variables (e.g. mean, standard deviation, standard error of the mean, number of patients, minimum, maximum), frequency tables, or ratios for discrete variables, and $95 \%$ confidence intervals. Statistical analyses were done by using software (Graph Pad Prism, Version 5.0) with repeated measures for student-t test to determine significant values at $\mathrm{p}<0.05$ level along with $\mathrm{r}$ (Pearson's correlation coefficient) values to determine strong and weak correlation among two variables for measuring different improvement parameters of combinedsex, female and male patients separately. The comparison was done between the experimental group and the control group. An alpha level of $5 \%$ was established i.e. a p-value less than 0.05 was considered statistically significant.

\section{Results}

\section{Enrolment and baseline characteristics of patients}

A total of 162 patients (72.22\% women) aged $59.89 \pm 7.19$ years those who were suffering with MD-OAD for $7.89 \pm 1.90$ years having massive muscular wasting specially in the thigh and calf regions, bone erosion and skeletal muscle damaged and 153 subjects aged $58.96 \pm 8.63$ years without MD-OAD confirmed by the serum tests of CK-MM and AldoA, electromyography, muscle biopsy, muscle ultrasound, heart-monitoring tests and radiological images (CT-scan or X-ray or MRI) included in the study. The patients under experimental group were suffering with severe intensity of pain, imbalanced quality of life, impaired joint and limb functions due to muscle wasting, weakness and degeneration and unable to perform daily activities confirmed by KOOS, KPS score, Katz ADL index, deranged anatomical features and radiological images. The baseline demographic characteristics of all subjects are shown in Table 1. The patients of the experimental group, suffering with MD-OAD, were not being treated by oral medications; injections and any type of alternative interventions or treatments for diminishing pain or inflammation, for muscle relaxation or for improvement of skeletal muscles one day prior to inclusion in the study and had not undergone arthrocentesis within four months prior to the blood tests such as CK-MM and AldoA evaluated at the baseline. The risk factors and values of the biochemical markers, such 


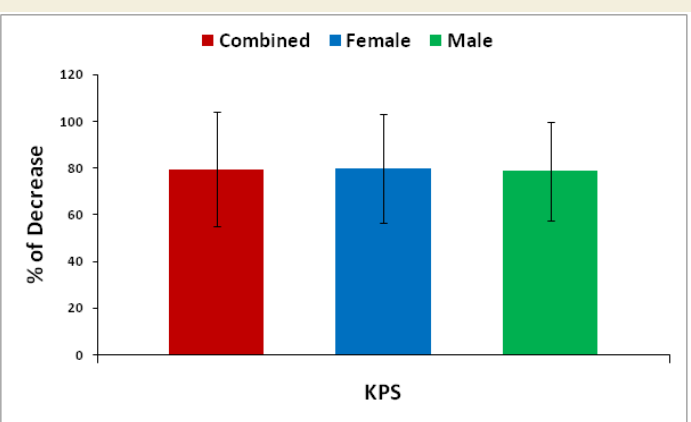

Figure 1: \% of decreased KPS over control subjects $\left({ }^{*} p<0.05\right)$

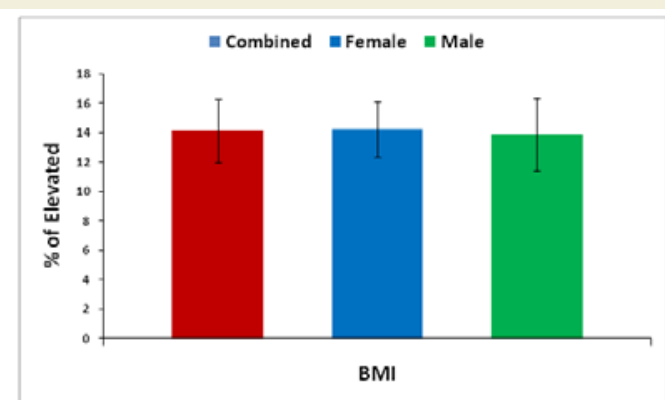

Figure 2: \% of elevated BMI over control subjects $\left({ }^{*} p<0.0001\right)$.

as CK-MM and AldoA were evaluated using the serum samples of experimental subjects, suffering with MD-OADs and control subjects, without MD-OADs.

\section{Biochemical parameters}

The biochemical parameters such as CK-MM and AldoA were measured and rechecked by the S-240 Mindray fully automated biochemistry analyzer before reporting the final test results for the subjects of experimental and control groups separately.

Table $2 \mathrm{~A}$ shows that the mean \pm SD levels of CK-MM and Aldo A for combined-sex, female-only and male-only patients with MDOADs were increased from their normal limits (CK-MM: for male $<171$ and female $<145 \mathrm{U} / \mathrm{L}$ and AldoA: $<7.6 \mathrm{U} / \mathrm{L}$ ) and their differences were highly significant $(\mathrm{p}<0.0001)$ when compared with the control subjects.

Moreover, Table 2B shows that the ratios of serum levels of CK$\mathrm{MM}$ and AldoA of experimental subjects with MD-OADs, (CK$\mathrm{MM}^{\mathrm{Exp}}$ : Aldo $A^{\mathrm{Exp}}$ ), and the control subjects without MD-OAD (CK$\mathrm{MM}^{\mathrm{Con}}$ : AldoA $\left.{ }^{\mathrm{Con}}\right)$ were highly significant $(\mathrm{p}<0.0001)$ when compared with the control subjects both overall and separately by gender.

Table 3 shows that Pearson's correlation coefficients between: CK-MM for experimental subjects with MD-OAD (CK-MM ${ }^{\text {Exp }}$ ) and for control subjects without MD-OAD (CK-MM ${ }^{\mathrm{Con}}$ ), AldoA for patients with MD-OAD (Aldo $A^{\text {Exp }}$ ) and subjects without MDOAD ( AldoA $^{\text {Con }}$, CK-MM for patients with MD-OAD (CK-MM ${ }^{\text {Exp }}$ ) and AldoA for subjects with MD-OAD (AldoA ${ }^{\text {Exp }}$ ) and CK-MM for experimental patients with MD-OAD (CK-MM ${ }^{\mathrm{Exp}}$ ) and AldoA for control subjects (Aldo ${ }^{\mathrm{Con}}$ ) were all exhibited highly significant differences $(\mathrm{p}<0.05)$ for both overall and separately by gender. The Pearson's correlation coefficients between CK-MM for patients without MD-OAD (CK-MM ${ }^{\mathrm{Con}}$ ) and AldoA for subjects without MDOAD $\left(\right.$ Aldo $\left.^{\text {Con }}\right)$, were not significant $(p=0.057, p=0.016$ and $p=0.942$ respectively) for combined-sex, female-only and male-only subjects.

\section{Anatomical parameters}

Table $4 \mathrm{~A}$ shows that the mean \pm SD values of SLR and KFS in supine, prone and standing positions were all decreased and that were increased for KES in supine, prone and standing positions for experimental cohorts with MD-OAD and observed to be all asymmetrical for both the legs and all the differences were highly significant $(p<0.0001)$ when that were compared with the control cohorts, without MD-OAD for both overall and separately by gender.

Table 4B shows the overall measurements of KGB, DAP, DBP and DCM of experimental cohorts were all increased to asymmetrical for both the legs and were all highly significant $(\mathrm{p}<0.05)$ when that were compared with the control subjects both overall and separately by gender. The overall measurements of DTM of experimental cohorts were all decreased and observed to be asymmetrical for both the legs when that were compared with the control subjects. The differences of mean \pm SD values of DTM were not significant for both right and left legs $(\mathrm{p}=0.485$ and $\mathrm{p}=0.486 ; \mathrm{p}=0.426$ and $\mathrm{p}=0.506 ; \mathrm{p}=0.647$ and 0.782 respectively) when that were compared with the control subjects both overall and separately by gender.

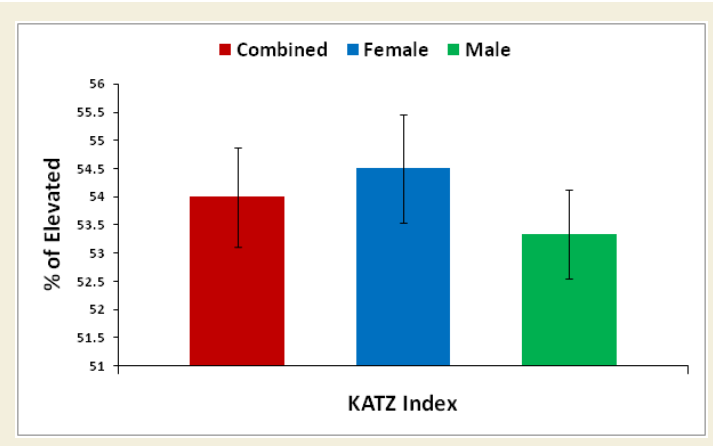

Figure 3: \% of elevated KATZ index over control subjects ( ${ }^{*} p<0.0001$ ).

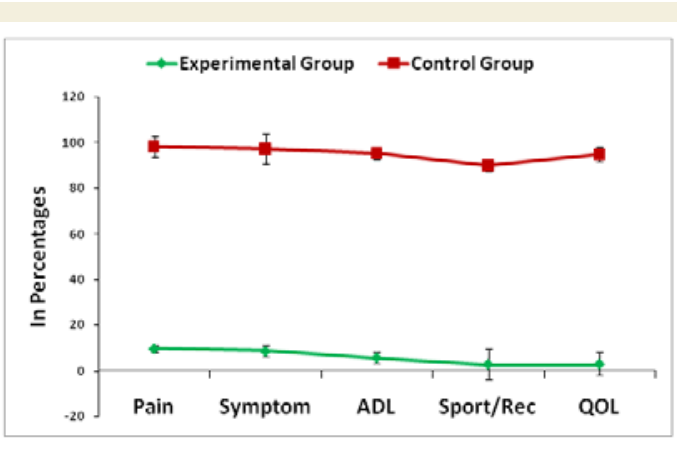

Figure 4: KOOS profiles of 163 subjects with MD-OAD and 153 subjects without MD-OAD ( $\left.{ }^{*} p<0.0001\right)$. 


\section{Independence in activities of daily living, performance status, knee pain related parameters and $\mathrm{BMI}$}

The percentage of reduction of independence in activities of daily living under Katz ADL index for MD, percentage of reduction on performance on daily activities under KPS scale, the reduction of five separated knee-injury osteoarthritis scored subscales under KOOS knee survey and increased body weight as assessed by BMI of all experimental subjects with MD-OAD were evaluated both overall and separately by gender and their differences were all highly significant $(\mathrm{p}<0.0001)$ when compared with the respective data of control subjects, without MD-OAD and shown in Figures 1-4.

\section{Discussion}

Here we describe and suggest the novel biochemical parameters such as CK-MM and AldoA, anatomical measurements and international approved pain related outcome scales along with scale of measurement of the functional status to perform activities of daily living independently in combination to detect suitable diagnostic protocol for MD-OAD on the studied patients, more easily and with minimum cost.

The level of serum CK-MM is found to be elevated from the standard value (for male $<171$ and female $<145 \mathrm{U} / \mathrm{L}$ ) in response to muscular dystrophy, connective tissue damage, etc. [22,23,54,55], and AldoA level increases from the standard value $<7.6 \mathrm{U} / \mathrm{L}$ due to skeletal muscle damages, polymyositis, dermatomyositis, muscular dystrophy and inflammatory muscle disease and bone erosion [56], suggesting that these markers may be risk factors for MD-OADs. Previous studies have utilized an individual research approach for different diseases [22,23,32-35] but have not studied in combined affect in the serum levels of these markers in MD-OADs.

The author has already established previously that the elevated levels of biomarkers such as C-Reactive Protein (CRP), CK-MM and AldoA represent the predictive risk factors such as inflammation, muscle degeneration, and skeletal muscle damage during OADs and also normalize their elevated levels with the help of specialized phytotherapeutic treatment protocol $[38,54,55]$. Therefore, the diagnostic and treatment protocol have already been established so far as only OADs are concerned but not in MD-OADs.

Moreover, the result shows that the Pearson's correlation coefficients (r-values) were highly predictive between the two biomarkers in patients with MD-OADs when they are separately compared with the values of healthy control subjects without MDOADs.

The author has further established the deranged anatomical features are the risk factors for OADs and also established the normalization of the deranged anatomical features for OADs with the help of established phytotherapy [33-35,57-59].

It is indicated from the present results that various anatomical parameters are deranged due to muscle wasting, weakness and degeneration, connective tissue damage, potential loss of joint cartilage, bone hypertrophy and joint effusion as a result of which both the legs become asymmetrical, when patient suffers with MD$\mathrm{OAD}$. When we further analyses the limb-wise deformities, we find that the KGBs are increased and not touching the back of knee joints (popliteal region) on the bed while supine and become asymmetrical due to the cumulative effects of muscle wasting, weakness, inflammation and stiffness of the connective muscles during the diseases condition as well as these further increased due to prolong use of knee supports, hyaluronic acid injections or corticosteroidal injections or arthrocentesis used for quick diminishing of pain, stiffness, inflammation and for increasing the strength of muscles temporally. There is increased in asymmetrical of DTM between the legs because of the cumulative effects of muscular wasting/ muscular bulging in the posterior region of the thighs and may be commonly compressed the sciatic nerve which is originating from the tubersity of the ischium and inserting to the tibia resulting which patient is complained the acute or mild pain in the lumbar region along with knee pain. The reason for decreased asymmetrical DCM of both legs is due to cumulative muscular wasting / stiffness of gastrocnemius muscles for which further compressed the tibial nerve which is a branch of sciatic nerve, may be affected of prolong use of knee supports, tenderness of Achilles tendon, soleus, calcaneus spurs, rigidity of ankle joints and such other reasons. Therefore, the slight difference of the diameter of the calf muscle of two legs trigger up the compression in the lumbar vertebrae as they misaligned. The asymmetry of DAP is the reason of cumulative effects of muscular wasting, inflammation, effusion or blood clotting due to engorgement of saphenous vein may be the extra reason of using knee braces, hyaluronic acid injections or corticosteroidal injections or arthrocentesis used for quick diminishing of pain, stiffness and inflammation and at the same time the condition of asymmetry for both the DBPs arise due to the cumulative effects of muscular wasting, inflammation, effusion or blood clotting on the anterior, posterior, lateral and medial parts of lower legs especially tibialis and anterior extensor hallucis longus and digitorial longus, gastrocnomius, and Achilles tendon.

Moreover, it is known that the knee flexion and extension are two main activities of the knee joint. All the muscles that move these joints are located in the anterior, posterior and lateral thigh region and for the flexion activity the muscles nerve root is sciatic nerve and for extension is femoral nerve. From the results, it reveals that SLR, KFS, KFP and KFSt are all reduced and at the same time the KES, KEP and KESt are all increased during MD-OAD because of massive muscular wasting, stiffness and muscle degeneration especially the quadriceps muscles and tendons, and healthy muscle fibers replaced by fibrosis and fat making muscle tissues in the thigh region and the legs become asymmetry. This phenomenon can be easily identified with the measurements of straight line rising, knee flexions and extensions of both the legs without going through any sophisticated tests.

Therefore, the measurements of the above-mentioned areas firmly indicate the condition of muscular wasting, weakness, degenerative status along with the condition of bone joints of the legs immediately without using the EMG, skeletal muscle ultrasound, MRI, CT-Scan and X-ray without any cost. Moreover, to identify the status of muscular wasting, weakness and degeneration especially skeletal muscles during $\mathrm{MD}-\mathrm{OAD}$ various muscles ought to be examined, but it is not at all possible to capture images or tests of multiple places with the help of EMG, CT-can, MRI, X-ray etc which are not only time taking but also expensive. Hence, in order to diagnose the MD$\mathrm{OAD}$, the measurements of anatomical parameters have been taken 
Citation: Ganguly A, Ganguly D. Muscular Dystrophy during Osteoarthritic Disorder: A Novel Diagnostic Protocol Evidenced by Biochemical and Anatomical Parameters. J Orthopedics Rheumatol. 2018; 5(2): 14.

care in addition to two biochemical tests.

Moreover, during MD-OAD, patients suffer with severe pain, stiffness of muscles, deteriorate the functional activities of daily living and the quality of life which can be easily identified with the help of international acclaimed scales such as KPS, KOOS and Katz ADL index as well as obesity is the another important criteria for MD$\mathrm{OAD}$ which can be measured by BMI.

Hence, when framing the novel protocol for diagnosis of $\mathrm{MD}$ $\mathrm{OAD}$, the above mentioned parameters are well taken care along with biochemical and anatomical features which are not at all possible with the help of radiological images or other established costly sophisticated tests.

Therefore, the above-mentioned studies are based on specific diagnosis for specific disease, which has several limitations such as costly diagnosis, target specific viz. muscles, tissues, bones, etc. and single parameter cannot be achieved to diagnose MD-OADs. The present study has an endeavor and first-time approach to critically diagnosis both the diseases such as muscular dystrophy and OAD in combination with the help of anatomical abnormalities, biochemical markers and muscle pain index along with KPS, KOOS, Katz $\mathrm{ADL}$ index and BMI indices. The combination of this approach is confirming muscular dystrophy during the progression of OADs, which are lacking in earlier studies.

\section{Conclusion}

Based on the present study, diagnostic protocol has been designed to detect MD-OAD by the abnormal characteristic features of elevated levels of serum CK-MM and AldoA, intensity of pain, imbalanced quality of life, impaired joint and limb functions due to muscle wasting, weakness and degeneration; therefore, monitoring the levels of serum CK-MM and AldoA along with anatomical parameters and international acclaimed scales such as KPS, KOOS, Katz ADL index and BMI may be a novel approach for detection of muscular dystrophy during OAD quickly and in affordable low cost, even at early progressive stage when there is no significant pain syndrome or discomfort or abnormalities observed in muscles other than expensive and time taken tests such as muscle biopsy, EMG, CT-can, skeletal muscle ultrasound, MRI. Future research should be carried out to detect the structural and functional abnormalities of Dystrophin protein in serum during MD-OADs.

\section{References}

1. Loeser RF, Goldring SR, Scanzello CR, Goldring MB (2012) Osteoarthritis: a disease of the joint as an organ. Arthritis Rheum 64: 1697-1707.

2. Felson DT, Lawrence RC, Dieppe PA, Hirsch R, Helmick CG, et al. (2000) Osteoarthritis: new insights. Part 1: The disease and its risk factors. Ann Intern Med 133: 635-646.

3. Bellayou H, Hamzi K, Rafai MA, Karkouri M, Slassi I, et al. (2009) Duchenne and Becker muscular dystrophy: contribution of a molecular and immunohistochemical analysis in diagnosis in Morocco. J Biomed Biotechnol 2009: 325210

4. Walsh MC, Hunter GR, Livingstone MB (2006) Sarcopenia in premenopausal and postmenopausal women with osteopenia, osteoporosis and normal bone mineral density. Osteoporos Int 17: 61-67.

5. Cruz-Jentoft AJ, Baeyens JP, Bauer JM, Boirie Y, Cederholm T, et al. (2010) Sarcopenia: European consensus on definition and diagnosis: report of the
European working group on sarcopenia in older people. Age Ageing 39: $412-$ 423.

6. Scott D, Blizzard L, Fell J, Jones G (2012) Prospective study of self-reported pain, radiographic osteoarthritis, sarcopenia progression, and falls risk in community-dwelling older adults. Arthritis Care Res (Hoboken) 64: 30-37.

7. Lang TF (2011) The bone-muscle relationship in men and women. J Osteoporosis 2011: pp. 4.

8. Frost HM (1987) Bone mass and the mechanostat: a proposal. Anat Rec 219: 1-9.

9. Frost HM (2000) Muscle, bone, and the Utah paradigm: a 1999 overview. Med Sci Sports Exerc 32: 911-917.

10. Brancaccio P, Lippi G, Maffulli N (2010) Biochemical markers of muscular damage. Clin Chem Lab Med 48: 757-767.

11. Dam EB, Loog M, Christiansen C, Byrjalsen I, Folkesson J, et al. (2009) Identification of progressors in osteoarthritis by combining biochemical and MRI-based markers. Arthritis Res Ther 11: R115.

12. Kane D, Grassi W, Sturrock R, Balint PV (2004) Musculoskeletal ultrasound-a state of the art review in rheumatology. Part 2: Clinical indications for musculoskeletal ultrasound in rheumatology. Rheumatology 43: 829-838.

13. Bureau NJ, Ali SS, Chhem RK, Cardinal E (1998) Ultrasound of musculoskeletal infections. Semin Musculoskelet Radiol 2: 299-306.

14. Bureau NJ, Cardinal E, Chhem RK (1998) Ultrasound of soft tissue masses. Semin Musculoskelet Radiol 2: 283-298.

15. Fornage BD (2000) The case for ultrasound of muscles and tendons. Semin Musculoskelet Radiol 4: 375-391.

16. Reimers CD, Finkenstaedt M (1997) Muscle imaging in inflammatory myopathies. Curr Opin Rheumatol 9: 475-485.

17. McDonnell CH, Jeffrey RB Jr, Bjorkengren AG, Li KC (1992) Intraarticular sonography for imaging the knee menisci: evaluation in cadaveric specimens. Am J Roentgenol 159: 573-574.

18. Patonay L, Nagy K, Engelke W (2002) Real-time endoarticular ultrasound imaging of the TMJ-a new diagnostic possibility? A cadaver study. Int J Oral Maxillofac Surg 31: 553-557.

19. Wakefield RJ, Brown AK, O'Connor PJ, Emery P (2003) Power doppler sonography: improving disease activity assessment in inflammatory musculoskeletal disease. Arthritis Rheum 48: 285-288.

20. D’Agostino MA, Said-Nahal R, Hacquard-Bouder C, Brasseur JL, Dougados M, et al. (2003) Assessment of peripheral enthesitis in the spondylarthropathies by ultrasonography combined with power Doppler: a cross-sectional study. Arthritis Rheum 48: 523-533.

21. Dannecker EA, Knoll V, Michael E, Robinson ME (2008) Sex differences in muscle pain: self-care behaviors and effects on daily activities. J Pain 9: 200209.

22. Omori G, Koga Y, Tanaka M, Nawata A, Watanabe H, et al. (2013) Quadriceps muscle strength and its relationship to radiographic knee osteoarthritis in Japanese elderly. J Orthop Sci 18: 536-542.

23. Felson DT (2006) Osteoarthritis of the knee. N Engl J Med 354: 841-848.

24. Ganguly A (2016) Normalization of varus/valgus deformities in osteoarthritis by external application of phytoconstituents: confirmed with anatomical observations and biochemical profiles and radiological images. Anat Physiol 6: 224

25. Ganguly A (2017a) Determination of the impact of functional instability of parathyroid hormone and the calcium-phosphorus ratio as risk factors during osteoarthritic disorders using receiver operating characteristic curves. Int Arch BioMed Clin Res 3: 39-46.

26. Ganguly A (2017b) Assessment of relationship between calcium-phosphorus ratio and parathyroid hormone levels in serum of osteoarthritic disordered patients: A diagnostic protocol. IOSR J Dent Med Sci 16: 46-54 
Citation: Ganguly A, Ganguly D. Muscular Dystrophy during Osteoarthritic Disorder: A Novel Diagnostic Protocol Evidenced by Biochemical and Anatomical Parameters. J Orthopedics Rheumatol. 2018; 5(2): 14

27. Slemenda C, Brandt KD, Heilman DK, Mazzuca S, Braunstein EM, et al. (1997) Quadriceps weakness and osteoarthritis of the knee. Ann Intern Med 127: $97-104$

28. Slemenda C, Heilman DK, Brandt KD, Katz BP, Mazzuca SA, et al. (1998) Reduced quadriceps strength relative to body weight: a risk factor for knee osteoarthritis in women? Arthritis Rheum 41: 1951-1959.

29. Liikavainio T, Lyytinen T, Tyrvainen E, Sipila S, Arokoski JP (2008) Physical function and properties of quadriceps femoris muscle in men with knee osteoarthritis. Arch Phys Med Rehabil 89: 2185-2194.

30. Palmieri-Smith RM, Thomas AC, Karvonen-Gutierrez C, Sowers MF (2010) Isometric quadriceps strength in women with mild, moderate, and severe knee osteoarthritis. Am J Phys Med Rehabil 89: 541-548.

31. Alnahdi AH, Zeni JA, Snyder-Mackler L (2012) Muscle impairments in patients with knee osteoarthritis. Sports Health 4: 284-294.

32. Oiestad BE, Juhl CB, Eitzen I, Thorlund JB (2015) Knee extensor muscle weakness is a risk factor for development of knee osteoarthritis. A systematic review and meta-analysis. Osteoarthritis Cartilage 23: 171-177.

33. Ganguly A (2015) Degenerative changes in lumbar region always lead to bilateral degenerative changes in knee-joints and vice-versa: sensation of pain cannot only be the parameter of degeneration. Anat Physol S4- 005.

34. Ganguly A (2017) Diagnosis, prevention \& phytotherapy for osteoarthritic disorders. Scholars Press Book, SIA OmniScriptum Publishing, European Union, pp: 84

35. Ganguly A (2018) Diagnosis of knee osteoarthritis risk through abnorma musculo-postural features. Int Arch BioMed Clin Res 4: 39-46.

36. Mikesky AE, Mazzuca SA, Brandt KD, Perkins SM, Damush T, et al. (2006) Effects of strength training on the incidence and progression of knee osteoarthritis. Arthritis Rheum 55: 690-699.

37. Ganguly A, Ganguly D, Banerjee SK (2018) Topical phytotherapeutic treatment: management of normalization of elevated levels of biochemical parameter during osteoarthritic disorders: A prospective study. J Orthopaedics Rheumatol 5: 14

38. Ganguly A, Ganguly D (2018) Topical phytotherapy: management of deranged anatomical and abnormal biochemical risk factors during failed total knee arthroplasty: a unique case study. J Orthopaedics Rheumatol 5: 6 .

39. Jan MH, Lai JS, Tsauo JY, Lien IN (1990) Isokinetic study of muscle strength in osteoarthritic knees of females. J Formos Med Assoc 89: 873-879.

40. Segal NA, Glass NA, Torner J, Yang M, Felson DT, et al. (2010) Quadriceps weakness predicts risk for knee joint space narrowing in women in the MOST cohort. Osteoarthritis Cartilage 18: 769-775.

41. Hortobagyi T, Garry J, Holbert D, Devita P (2004) Aberrations in the control of quadriceps muscle force in patients with knee osteoarthritis. Arthritis Rheum 51: $562-569$

42. O'Reilly SC, Jones A, Muir KR, Doherty M (1998) Quadriceps weakness in knee osteoarthritis: the effect on pain and disability. Ann Rheum Dis 57: 588 594
43. Sanchez-Ramirez DC, van der Leeden M, van der Esch $M$, Gerritsen M, Roorda LD, et al. (2013) Association of serum C-reactive protein and erythrocyte sedimentation rate with muscle strength in patients with knee osteoarthritis. Rheumatology (Oxford) 52: 727-732.

44. Marks R (2015) Muscle and muscle mechanisms as possible factors leading to osteoarthritis. SM J Orthop 1: 1008.

45. Katz JN, Chang LC, Sangha O, Fossel AH, Bates DW (1996) Can co-morbidity be measured by questionnaire rather than medical record review? Med Care 34: 73-84.

46. Singh B, Bhaya M, Stern J, Roland JT, Zimbler M, et al. (1997) Validation of the Charlson co-morbidity index in patients with head and neck cancer: a multi-institutional study. Laryngoscope 107: 1469-1475.

47. Cabaniss C (1990) The History, Physical, and Laboratory Examinations. In Walker HK, Hall WD, et al. (Eds), Clinical Methods ( $\left.3^{\text {rd }} e d n\right)$, Butterworths, Boston, USA, pp. 161-163.

48. Feissli S (1966) Quantitative determination of Aldolase. KlinWschr 44: 390

49. American Academy of Orthopaedic Surgeons (AAOS) (1965) Joint motion: method of measuring and recording. The Academy, Chicago, USA, pp. 87.

50. Katz S, Down TD, Cash HR, Grotz RC (1970) Progress in the development of the index of ADL. Gerontologist 10: 20-30.

51. Schag CC, Heinrich RL, Ganz PA (1984) Karnofsky performance status revisited: reliability, validity, and guidelines. J Clin Oncol 2: 187-193.

52. Roos EM, Lohmander LS (2003) The Knee injury and Osteoarthritis Outcome Score (KOOS): From joint injury to osteoarthritis. Health Qual Life Outcomes 1: 64 .

53. Ferrera LA (2006) Focus on body mass index and health research. Nova Science Publishers Inc. New York, USA, pp. 1-27.

54. Ganguly A (2018) Levels of C-reactive protein, creatine kinase-muscle and aldolase $\mathrm{A}$ are suitable biomarkers for detecting risk factors for osteoarthritic disorders: a novel diagnostic protocol. Caspian J Intern Med.

55. Ganguly A (2018) Role of topical phytotherapeutic effects on elevated leve of serum creatine kinase-muscle as risk factor of muscular degeneration in knee-osteoarthritis: Part III. A systematic meta-analysis. Int Arch BioMed Clin Res.

56. Ganguly A (2018) Impact of tropical phytotherapy on the risk factor of elevated level of serum Aldolase-A in knee-osteoarthritis: Part II. A systematic metaanalysis. Int Arch BioMed Clin Res.

57. Ganguly A (2015) Degenerative changes in lumbar region always lead to bilateral degenerative changes in knee-joints and vice-versa: sensation of pain cannot only be the parameter of degeneration. Anat Physol S4-005.

58. Ganguly A (2014) Tropical phytotherapeutic treatment for achieving knee symmetry in osteoarthritis - A sustainable approach. Int J Phytomed 6: 489509.

59. Ganguly A (2015) Obtaining normal flexion and extension of knee joints on supine, prone and standing positions in osteoarthritis by topical phytotherapeutic treatment irrespective of age and sex. Int J Phytomed 7: 290-301.

\section{Acknowledgement}

The author acknowledges the assistance of Ayondeep Ganguly and Anondeeep Ganguly for coordinating patients and arranging all data from the biochemical analysis. The author also thanks Dr. Swapan Roy, Chief Biochemist at the Galaxy Medical Centre in Kolkata, India, for analyzing the biochemical parameters in the present study. 